\title{
Técnica de impresión para rebordes móviles. Parte 2: Mandibula.
}

\section{Impression technique of mobile fibrous ridge. Part 2:Jaw.}

\begin{abstract}
1. Unidad de Prótesis Removible. Servicio de Sanidad Dental, Sub Dirección Odontológica, Hospital de Carabineros HOSCAR. Chile
\end{abstract}

* Correspondencia autor. Felipe San Martín Forray. Dirección: Hospital de Carabineros, Antonio Varas 2500, clínica $18,3^{\circ}$ piso .Edificio Corporativo, Ñuñoa, Santiago, Chile. | Teléfono: +5999698 0277 - +562 22441172 | E-mail:ghpaisaje@gmail. com

Trabajo recibido el 21/05/2018.

Aprobado para su publicación el 12/08/2018

\author{
Felipe San Martín - Forray ${ }^{1 *}$
}

\section{RESUMEN}

El reborde fibroso móvil mandibular -producto de reabsorción alveolar extrema, trauma oclusal o inestabilidad protésica - se deforma bajo presión, alterando la retención, soporte y estabilidad protésica. La impresión debe registrarlo en forma pasiva, evitando comprimirlo y desplazarlo, transfiriendo la presión hidráulica del material de impresión a la zona de soporte útil del reborde. Se presenta una alternativa simplificada de impresión para reborde móvil mandibular. Se discuten sus ventajas y se contrasta con la literatura relacionada.

PALABRAS CLAVE:

Reborde móvil; Impresión mucoestática; Mandíbula; Estabilidad.

Rev. Clin. Periodoncia Implantol. Rehabil. Oral Vol. 12(2); 93-95, 2019.

\section{ABSTRACT}

The mandibular mobile fibrous ridge -product of extreme bone resorption, occlusal trauma or prosthetic instability - deforms under pressure, altering prosthetic retention, support and stability. The impression must register it passively; avoiding compression and movement, transferring the hydraulic pressure of the impression material to usable support area of the ridge. Presenting a simple alternative for mandibular mobile ridge impression .Its advantages are discussed and contrasted with the related literature.

\section{KEY WORDS:}

Mobile fibrous ridge; Mucostatic impression; Jaw; Stability.

Rev. Clin. Periodoncia Implantol. Rehabil. Oral Vol. 12(2); 93-95, 2019

\section{INTRODUCCIÓN}

El reborde fibroso móvil (RM) - provocado por reabsorción alveolar extrema, trauma oclusal o inestabilidad protésica, entre otras causasafecta la retención y estabilidad de la prótesis completa, ya que su deformación y desplazamiento en función desaloja el aparato(1-2-3).Su prevalencia en mandíbula es de $5 \%$, comprometiendo generalmente la región anterior del reborde, disminuyendo así el área útil de soporte ${ }^{(4)}$.

Descartada la cirugía preprotética de remoción del tejido, la impresión a realizar en la rehabilitación de estos casos debe ser a presión selectiva: mucoestática sobre el RM- a fin de no deformarlo- y mucocompresiva sobre la mayor área de soporte disponible, con objetivo de optimizar la estabilidad protésica ${ }^{(1-2-3-4-5)}$

Múltiples técnicas de impresión para RM han sido propuestas para el maxilar - ya que su prevalencia es mayor $(24 \%)^{(4)(6)}$ - pero pocas para la mandíbula(1). Se han descrito técnicas con cubetas individuales abiertas, perforadas, con "ventanas", articuladas o unidas con pines e imanes; junto con el uso de diversos materiales de impresión: yeso parís, hidrocoloide irreversible, siliconas de diferentes densidades, compuesto de modelar, ceras y pasta zinquenólica ${ }^{(4-7-8-9)}$. Algunas son muy exigentes en la operatoria clínica, dificultando el control de la presión sobre el RM, ya que comprenden varios pasos y combinación de materiales de impresión; generando potenciales distorsiones imperceptibles en el momento, pero que se manifiestan posteriormente en el deficiente desempeño funcional de la prótesis completa mandibular ${ }^{(2)}$.

Este reporte clínico presenta una nueva alternativa simplificada de impresión para reborde móvil mandibular.

\section{DESCRIPCIÓN DE CASO}

Sujeto de 78 años, ASA 1, desdentado total bimaxilar. Portador de prótesis completas desajustadas por cinco años. Presenta reborde óseo mandibular deprimido -tipo 5-6 de Atwood (Cawood y Howell)- recubierto por mucosa sana y fino RM, entre zonas de piezas 3.5 y 4.5 (Fig.1). Al presentar el sujeto una mucosa sana, no es necesario efectuar acondicionamiento de tejidos previo al tratamiento. Se planifica confección de nuevo juego de prótesis completas. Es notificado del tratamiento a realizar, proporciona su consentimiento informado del Hospital de Carabineros de Santiago, Chile y autoriza la toma de fotografías.

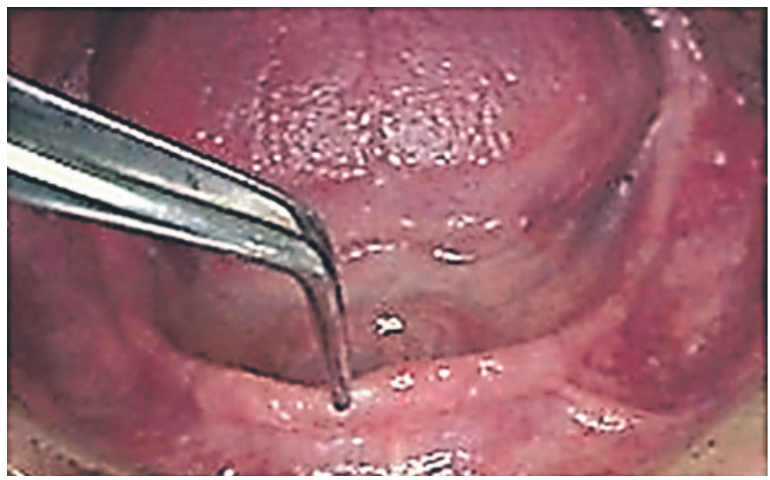

Figura 1. Paciente desdentado total bimaxilar. Mandíbula presenta gran reabsorción alveolar y fino reborde móvil fibroso en zona anterior

La impresión preliminar se realiza mediante cubeta de stock e hidrocoloide irreversible (Jeltrate, USA) de consistencia normal. Con bisturí se espacia la zona de RM y se rebasa con hidrocoloide irreversible de consistencia fluida. 
Sobre el modelo de estudio se delimita y cubre la zona de RM con dos láminas de cera a fin de espaciar y se confecciona una cubeta individual acrílica (Marché, Chile) con mango recto en la zona media lingual y una "ventana" sobre el área de RM (Fig.2 a).Sus bordes son ajustados en boca -con fresón de carbide- a la zona marginal neutra vestibular y al límite superior de las inserciones musculares del piso de boca. La cubeta cubre completamente ambas papilas retromolares. Ya recortada, se verifica que la cubeta no se desplace debido a la actividad muscular paraprotética. Mediante compuesto de modelar (Kerr, USA) se impresiona la zona de sellado principal: las papilas retromolares y todo el borde lingual, delimitado por movimientos linguales autogenerados por el paciente y guiados por el operador (Fig.2 b). No se aplica compuesto de modelar sobre el borde vestibular.
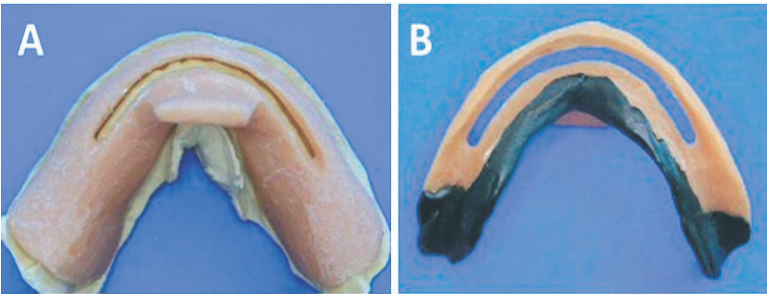

Figura 2. a: Cubeta individual con mango central y ventana espaciada sobre zona de reborde móvil. Cobertura completa de papilas retromolares. Se debe recortar y delimitar en boca. b: Cubeta delimitada. Impresión con compuesto de modelar de la zona principal de sellado

Se pincela la cubeta con adhesivo para prótesis (Coltene, Suiza) y se carga la zona posterior y lingual con silicona de polivinilsiloxano de adición (3M, USA) a través de pistola ; se asienta y estabiliza en boca mediante el mango central (Fig.3 a).Se introduce la punta aplicadora en la ventana y se colma con silicona todo el espaciado sobre el RM (Fig 3 b).Se completa el relleno con silicona por todo el borde vestibular (Fig. $3 \mathrm{c}$ ).Durante el tiempo de trabajo del material se combinan movimientos linguales autogenerados y movimientos de labios y mejillas guiados por el operador a fin de registrar la actividad neuromuscular.
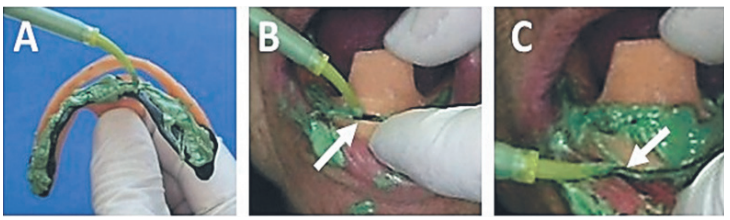

Figura 3. a: Carga de silicona en zona lingual y posterior de la cubeta. b: Relleno de la zona de reborde móvil a través de la ventana (flecha).c:Relleno del borde vestibular (flecha).

Se retira la cubeta de boca, se lava, se critica la impresión (Fig.4), se reposiciona en boca para verificar su retención y estabilidad. Se lava y desinfecta, se encofra y se obtiene el modelo de trabajo en yeso.

El juego de prótesis se termina de manera convencional, diseñando un esquema oclusal balanceado bilateral con leve inoclusión anterior. Se instala en boca y se controla regularmente.

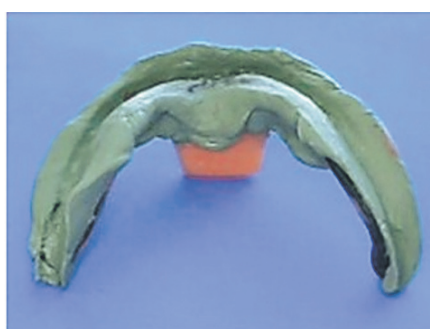

Figura 4. Impresión terminada.

\section{DISCUSIÓN}

El reborde móvil (RM) mandibular se caracteriza por una fina brida de tejido fibroso que cubre el reborde anterior, disminuyendo el área útil de soporte; dificultando su manejo ya que se deforma bajo presión.

La impresión preliminar con hidrocoloide irreversible de consistencia normal, espaciado y luego rebase de consistencia fluida disminuye la presión hidráulica sobre el RM optimizando la fidelidad del modelo de estudio $^{(12)}$ (ver Parte $\left.1^{(6)}\right)$.
El diseño de la cubeta individual comprende un mango vertical en la línea media lingual a fin de favorecer su prehensión y estabilidad, distribuyendo uniformemente la presión de asentamiento, sin entorpecer los movimientos musculares ni la visualización del RM al momento del relleno con silicona. El espaciado con cera y la ventana permiten introducir la silicona y disipar la presión hidráulica del material. Una vez recortada la cubeta, se verifica su estabilidad bajo movimientos de lengua, labios y mejillas; lo que indica que se encuentra asentada sobre la "zona neutra": espacio virtual de equilibrio de fuerzas neuromusculares que no desplazarán la futura prótesis en función ${ }^{(13)}$.

La impresión con compuesto de modelar se realiza sobre las papilas retromolares y el flanco lingual - áreas de sellado principal - por ser susceptibles de entrada de aire y pérdida del sellado. No se efectúa sobre el flanco vestibular ya que por la consistencia pesada del material, puede sobreextender el borde protésico y comprimir el RM. Estas delimitaciones están basadas en los postulados de Krol y Jacobson, quienes definen áreas de soporte y sellado sobre la anatomía remanente del desdentado total, a fin de mejorar la estabilidad protésica, preservando el remanente biológico(5-10-11).

La impresión con silicona registra primeramente las zonas de soporte posterior y lingual. Una vez asentada la cubeta, se rellena el espaciado a través de la ventana, permitiendo el escurrimiento del exceso de material, disipando la presión hidráulica sobre el RM. El complemento de relleno por el borde vestibular y los movimientos funcionales de labios y mejillas definen la extensión y la forma del flanco vestibular. Los movimientos linguales definen la extensión y forma del flanco lingual, registrando la posición más alta del piso de boca y frenillo lingual.

Se obtiene así en un solo tiempo operatorio, una impresión a presión selectiva: mucoestática en la zona de RM y mucocompresiva en la zona del reborde posterior y papilas retromolares. Es una técnica rápida, cómoda para el paciente y el operador, utiliza materiales de fácil manipulación y de bajo costo.

Difiere de la técnica maxilar (ver Parte $\left.1^{(6)}\right)$, ya que las características anatómicas y funcionales son distintas:

- El remanente óseo maxilar disponible es mayor, al poder aprovechar la superficie palatina. En la mandíbula el reborde óseo es escaso, debido a la avanzada reabsorción.

- La influencia de la musculatura paraprotética y lingual en la estabilidad protésica es mayor en la mandíbula que en el maxilar.

- Generalmente el RM maxilar es grueso, en tanto el RM mandibular es fino y más susceptible de deformarse.

- La cubeta mandibular precisa de un mayor espaciado y la ventana para disipar la presión hidráulica del material de impresión ejercida sobre el fino RM, evitando su distorsión.

- La cubeta maxilar debe contener el material y evitar su escurrimiento por gravedad. En la impresión mandibular, no es necesario, ya que esta fuerza favorece el asentamiento y distribución del material.

Ali et al ${ }^{(1)}$ proponen una técnica de cubeta individual con ventana, combinando registros en cuatro etapas con compuesto de modelar, ceras, pasta zinquenólica e inyección de yeso parís; complicando la operatoria y arriesgando distorsionar el registro del RM. Gupta et $\mathrm{al}^{(3)}$ presentan una cubeta con ventana y utilizan compuesto de modelar, pasta zinquenólica y yeso parís aplicado con pincel sobre RM. Pai et al ${ }^{(8)}$ utilizan una cubeta individual mutiperforada, compuesto de modelar y silicona de consistencia mediana en un tiempo operatorio. Se dificulta el control de la presión hidráulica del material sobre el RM ya que esta depende del número, diámetro y distribución de los orificios; así como de la consistencia de la silicona. Shah et $a^{(9)}$ realizan impresiones preliminares con compuesto de modelar. Sobre los modelos de estudio confeccionan cubetas individuales articuladas de dos tipos: dos piezas unidas por pines o por imanes. La impresión definitiva se obtiene mediante compuesto de modelar e inyección de pasta zinquenólica uniendo ambas piezas de las cubetas en boca durante el tiempo de trabajo de los materiales. Es una técnica muy compleja para el operador y técnico dental, con alta probabilidad de distorsión del registro.

El esquema oclusal balanceado bilateral con leve inoclusión anterior permite estabilizar la prótesis durante las trayectorias excursivas. Transfiere la carga oclusal a la zona premolar y molar, aliviando la zona del RM y evitando su deformación y desplazamiento.

\section{CONCLUSIÓN}

La técnica de impresión para reborde móvil mandibular descrita es simple, rápida, utiliza materiales de fácil manipulación y bajo costo; y registra el RM en forma pasiva sin deformarlo.

\section{CONFLICTO DE INTERÉS}

El autor declara no tener conflicto de interés de ninguna índole. 


\section{Bibliografía}

1. Ali M, Chaturvedi S, Shah M. Innovative tunnel technique for mandibular flabby ridge. Int J Dent Clin. 2012;4(2):74-76.

2. Lynch $\mathrm{C}$, Allen $\mathrm{P}$. Management or the flabby ridge: using contemporary materials to solve an old problem. Br Dent J. 2006;200(5):258-61.

3. Gupta S, Jain S, Berry R. Displaceable tissue: a clinical challenge for prosthodontist. Asian Pac J Health Sci. 2014;1(2):61-64.

4. Crawford R, Walmsley A. A review of prosthodontic management of fibrous ridges. Br Dent J. 2005;199:715-19.

5. Jacobson TE, Krol AJ. A contemporary review of the factors involved in completes dentures. Part III: support. J Prosthet Dent. 1983;49(3):306-13.

6. San Martín-Forray F. Técnica de impresión para rebordes móviles. Parte 1: maxilar. Rev Clin Periodoncia Implantol Rehabil Oral. 2017;10(3):173-175.

7. Shetty S, Nag PV, Shenoy KK. The selective pressure maxillary impression. A review of techniques and presentation of an alternate custom tray design. J Indian Prosthodont Soc. 2007;7(1):8-11.
8. Pai UY, Reddy VS, Hosi RN. A single step impression technique of flabby ridges using monophase polyvinylsiloxane material: a case report. Case Rep Dent. 2014;2014:104541.

9. Shah K, Parikh P, Naveen YG, Chabra T. Two alternatives for impression making of mandibular flabby ridge:case reports. Adv Hum Biol. 2013;3(2):37-41.

10. Jacobson TE, Krol AJ.A contemporary review of the factors involved in completes dentures. Part I: retention. J Prosthet Dent. 1983;49(1):5-15.

11. Jacobson TE, Krol AJ.A contemporary review of the factors involved in completes dentures. Part II: stability. J Prosthet Dent. 1983;49(2):165-72

12. Kitamura A, Kawai Y. Basic investigation of the laminated alginate impression technique: setting time, permanent deformation, elastic deformation, consistency and tensile bond strength tests. J Prosthodont Res. 2015;59:49-54.

13. Beresin V, Schiesser F. The neutral zone in complete dentures. J Prosthet Dent. 2006;95(2):93-100. 